\title{
Utilization of chitosan-coated superparamagnetic iron oxide nanoparticles for chromium removal
}

\author{
Antony V. Samrot ${ }^{1} \cdot$ N. Shobana ${ }^{1} \cdot$ P. Durga Sruthi ${ }^{1} \cdot$ Chamarthy Sai Sahithya $^{1}$
}

Received: 15 May 2018 / Accepted: 2 October 2018 / Published online: 9 October 2018

(c) The Author(s) 2018

\begin{abstract}
Superparamagnetic iron oxide nanoparticles (SPIONs) have been widely used for their versatility, while it is coated with a biopolymer like chitosan that adds attraction and also increases its applications. In this study, SPION was synthesized by chemical co-precipitation method, characterized using various analytical techniques like UV-Vis, FTIR, SEM, EDX, TEM, AFM, XRD, zeta potential and Raman spectroscopy analysis. Chitosan was coated onto the SPIONs and used for water treatment to remove chromium (450 ppm concentration). Chitosan-coated SPIONs were found to remove about $80 \%$ of chromium. Freundlich model was found to be fitting better for the current study.
\end{abstract}

Keywords Chitosan $\cdot$ SPIONs $\cdot$ Metal removal $\cdot$ Characterization $\cdot$ Chromium

\section{Introduction}

In today's world, alarming water pollution has become a major threat to the environment and has led to the development of new technologies to minimize the potential contaminants from the water bodies. Effluents from the mining industries, power generating industries, electronic industries and tanneries contain large amounts of heavy metals that pose a great risk to the surviving population in and around the water bodies (Liu et al. 2012) and cause bioaccumulation in beings that ingest these waters directly or indirectly (Yousafzai et al. 2017). Heavy metals such as uranium, mercury, chromium, arsenic, lanthanum, lead, cadmium and zinc are few of the commonly found metals that contaminate the water bodies (Boddu et al. 2003; Tchounwou et al. 2012). Chromium ( $\mathrm{Cr}, Z=24, A=51.9961 \mathrm{u} \pm 0.0006 \mathrm{u}$ ) is said to be relatively stable among the heavy metal ions of the periodic table and exists in several ionic forms. It is toxic and it is considered to be one of the major carcinogens as well as a mutagenic compound (Geng et al. 2009; Dayan and Paine 2001; Aitio et al. 1988). Thus, removal of this hazardous

Antony V. Samrot

antonysamrot@gmail.com

1 Department of Biotechnology, School of Bio and Chemical Engineering, Sathyabama Institute of Science and Technology, Chennai, Tamil Nadu 600119, India heavy metal has become a major goal and research interest among several researchers and led to the development of diverse methods. Although several methods have been devised, bio-sorption using bio-based particles/nanoparticles has been considered highly efficient (Lasheen et al. 2013; Sheet et al. 2014; Abdel-Raouf and Abdul-Raheim 2017; Mane et al. 2011; Ahluwalia and Goyal 2007) due to the large surface-to-volume ratio of nanoparticles compared to the bulk materials (Rajput et al. 2016). Using SPIONs is the better option, since superparamagnetic iron oxide nanoparticles (SPIONs) obey to external magnetic field; thus, it is easier to remove after the adsorption process (Gill et al. 2017) and also it is low toxic and non-reactive to human and majorly it has very strong adsorption capacity (Lasheen et al. 2013). Further, crystallinity, dispersion, size and shape also play an important role in the removal of heavy metals efficiently. One of the major challenges of aggregation faced by SPIONs can be overcome by surface modification or by following up certain parameters (coating a sorbent material) that result in the formation of monodispersed SPIONs (Justin et al. 2018; Mahmoudi et al. 2011). 
In order to increase the chemisorption efficiency of SPIONs, biopolymers such as chitosan can be used to coat the nanoparticles. Chitosan is an abundant biodegradable biopolymer available in the nature, which can be extracted from the exoskeletons of marine organisms such as crabs, lobsters, shrimps as well as fungi. Literature studies have suggested that chitosan is more efficient than its precursor chitin (Boddu et al. 2003). The free amine groups present on the surface of chitosan aid in the chemisorption of the metal ions and subsequently result in their removal from wastewater (Kaveeshwar et al. 2018). The coating of chitosan on SPIONs makes it less toxic to the environment resulting in an eco-friendly method of heavy metal removal. This use of nanoparticles in this method is highly cost-effective process (Gill et al. 2017) and hence can be executed in a large-scale clean-up processes. In this study, SPIONs were synthesized by chemical method, coated with chitosan and utilized for chromium removal from water.

\section{Materials and methods}

\section{Materials}

All chemicals used in this work were of analytical grade. Ferrous sulphate and ferric sulphate were procured from M/s Merck, India. Ammonia solution, tetramethyl ammonium hydroxide solution (TMAOH) and chitosan were obtained from SRL, India; acetic acid was purchased from Fisher Chemical, India. Potassium dichromate was obtained from Rankem, India.

\section{Synthesis of superparamagnetic iron oxide nanoparticles (SPIONs)}

SPIONs were synthesized by following chemical co-precipitation method. The synthesis procedure is as follows: $0.91 \mathrm{~g}$ ferrous sulphate $\left(\mathrm{FeSO}_{4}\right)$ and $3.2 \mathrm{~g}$ ferric sulphate $\left(\mathrm{Fe}_{2}\left(\mathrm{SO}_{4}\right)_{3}\right)$ were taken in $8 \mathrm{ml}$ and $2 \mathrm{ml}$ nitrogenated Milli$\mathrm{Q}$ water, respectively, and stirred well. The two solutions were mixed together while manually stirring it for $15 \mathrm{~min}$. $15 \mathrm{ml}$ of $25 \%$ ammonia solution was added dropwise to the precursor solution under constant vigorous stirring, and $15 \mathrm{ml}$ of $25 \%$ TMAOH was also added dropwise to the solution. As soon as the colour of the solution turned black, the addition of ammonia and TMAOH was stopped. The nanoparticles were allowed to settle using a magnet, and excess reducing agent was pipetted out. Nanoparticles were washed several times with Milli-Q water until the $\mathrm{pH}$ dropped to neutral. Further, the nanoparticles were lyophilized in vacuum to obtain powder particles (Eom et al. 2010).

\section{Preparation of chitosan-coated SPIONs}

Chitosan-coated SPIONs were prepared according to the protocol followed by Sureshkumar et al. (2016) with some modifications. $100 \mathrm{mg}$ of chitosan was added to $40 \%$ acetic acid and stirred for $24 \mathrm{~h}$. Then, $100 \mathrm{mg}$ of SPIONs was added and was sonicated for $30 \mathrm{~min}$. The sample was then lyophilized in vacuum.

\section{Characterization of SPIONs}

The prepared SPIONs were characterized for further studies. Absorbance spectrum of SPIONs was recorded at UV-Vis spectrometer. Fourier transform infrared spectroscopy (FTIR) of prepared SPIONs was analysed using IR Affinity-1s, Shimadzu, Japan, for wave number range of $4000-400 \mathrm{~cm}^{-1}$. Microscopic analysis was investigated using transmission electron microscope (TEM) (TEECNAI G2 Spirit Biotwin-120 kV) and atomic force microscopy (AFM) (Bruker, Germany). X-ray diffraction (XRD) patterns of the SPIONs were recorded by Smartlab X-ray diffractometer (Rigaku, Japan). The XRD patterns were taken in the $2 \theta$ range of $20^{\circ}-80^{\circ}$ in a fixed-time mode at room temperature. To determine the stability of the SPIONs, zeta potential value was recorded (Brookhaven ZetaPALS). Raman spectroscopy was also performed using LabRam HR 800 model of Horiba Jobinyvon.

\section{Chromium metal removal}

Adsorption studies were carried out according to the procedure quoted by Sureshkumar et al. (2016) with some modifications. $5 \mathrm{mM}$ potassium dichromate was dissolved in $90 \mathrm{ml}$ of distilled water which contains $450 \mathrm{ppm}$ of chromium metal $\mathrm{Cr}(\mathrm{VI})$ and was divided into three portions in which $10 \mathrm{mg} / 10 \mathrm{ml}, 20 \mathrm{mg} / 10 \mathrm{ml}$ and $30 \mathrm{mg} / 10 \mathrm{ml}$ of chitosan-coated SPIONs in distilled water were added in each portion of solution separately. It was stirred continuously at $400 \mathrm{rpm}$ at $22{ }^{\circ} \mathrm{C}$ for $3 \mathrm{~h}$, and the $\mathrm{pH}$ was maintained acidic at 5.7 (Anah and Astrini 2017; Namasivayam and Ranganathan 1993). $1 \mathrm{ml}$ of sample was withdrawn from each solution every $15 \mathrm{~min}$ for about $3 \mathrm{~h}$. Chitosancoated SPIONs were made to settle under the magnetic field, and the solution above the settled residue was then analysed for absorbance at $350 \mathrm{~nm}$ (Shimadzu, Japan). The concentration of the chromium was determined against the standard graph and \% removal was determined by the following formula:

$\%$ removal $=\frac{C_{0}-C_{t}}{C_{0}} \times 100$ 
To determine the adsorbent's ability and other constants, Langmuir isotherm (Kumar and Sivanesan 2006), Freundlich isotherm (Dada et al. 2012) and Temkin isotherm (Piccin et al. 2011) were carried.

\section{Results and discussion}

\section{Characterization of SPIONs and chitosan-coated SPIONs}

$\mathrm{UV}-\mathrm{Vis}$ analysis was performed and their respective peaks near $225 \mathrm{~nm}$ and $360 \mathrm{~nm}$ indicated the synthesis of SPIONs and chitosan-coated SPIONs (Fig. 1a, b), where
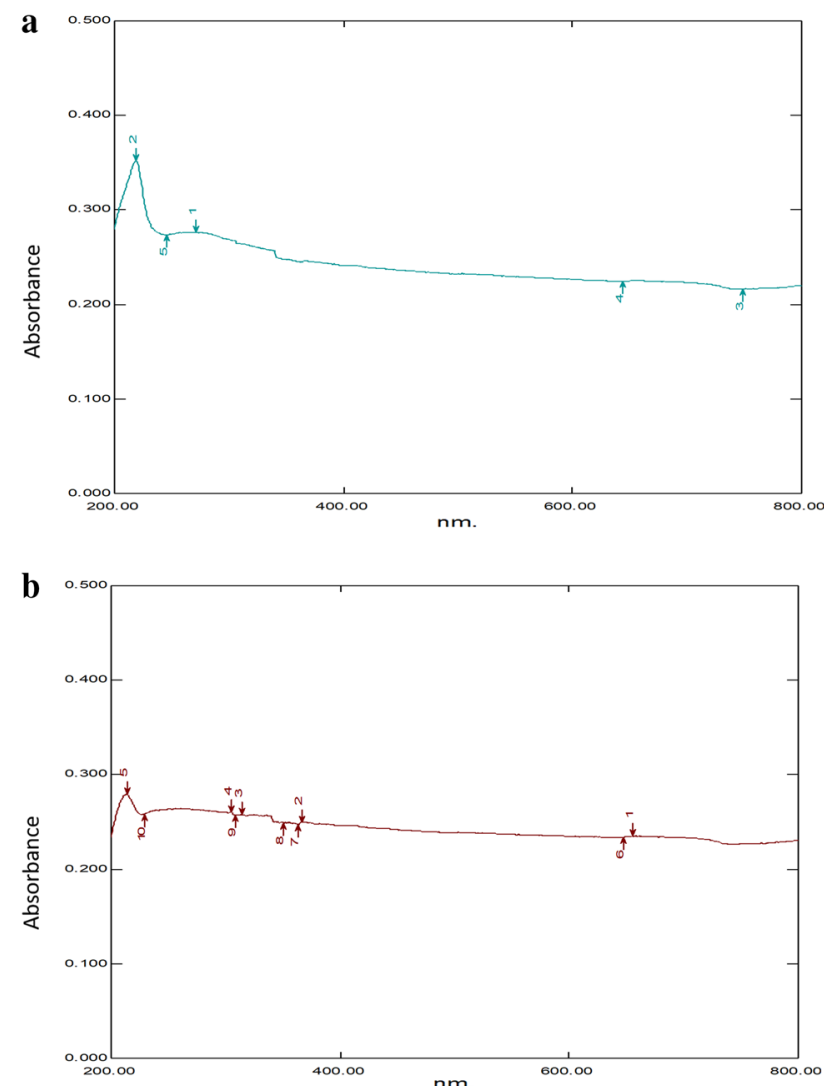

Fig. 1 UV-Vis analysis of a SPIONs, b chitosan-coated SPIONs

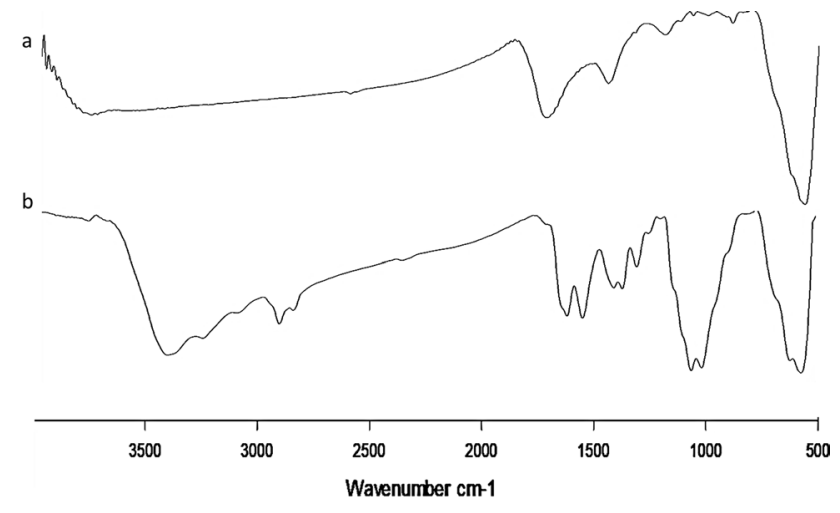

Fig. 2 FTIR analysis of a SPIONs, b chitosan-coated SPIONs

Samrot et al. (2017) also found magnetite nanoparticles to have absorbance maximum around $230-250 \mathrm{~nm}$. In FTIR, the presence of strong absorption bands at around 569 and $575 \mathrm{~cm}^{-1}$ showed the formation of magnetic nanoparticles $(\mathrm{Fe}-\mathrm{O}$ stretching vibration of tetrahedral sites of spinel structure) (Fig. 2a, b) (Inbaraj and Chen 2012). The peak at $2920 \mathrm{~cm}^{-1}, 1627 \mathrm{~cm}^{-1}, 1416 \mathrm{~cm}^{-1}$ and $1069 \mathrm{~cm}^{-1}$ was the stretching vibrations of $-\mathrm{CH}$, $\mathrm{N}-\mathrm{H}, \mathrm{C}-\mathrm{N}$ vibration of amino group and $\mathrm{C}-\mathrm{O}$ in the ether group of chitosan, respectively (Fig. 2b). TEM analysis of both SPIONs and chitosan-coated SPIONs showed it is spherical in shape (Fig. 3a, b). AFM analysis of SPIONs showed that the nanoparticles were well dispersed and size below $20 \mathrm{~nm}$ (Fig. 4a) and below $35 \mathrm{~nm}$ in case of chitosan-coated SPIONs (Fig. 4b). The SPIONs and chitosan-coated SPIONs were crystalline in nature as they have shown reflection pattern in XRD at 220, 311, 400, 511 and 440, and the shape was cubic spinel structure of $\mathrm{Fe}_{3} \mathrm{O}_{4}$ (JCPDS 85-1436) (Marquez et al. 2012) (Fig. 5a, b). The zeta potential value of the SPIONs was found to be $-38.10 \mathrm{mV}$ and that of chitosan-coated SPIONs to be $3.34 \mathrm{mV}$ suggesting that the SPIONs were stable than the latter (Figs. 6a, b) (Bhattacharjee 2016). In Raman spectroscopy, the minor peak at $600 \mathrm{~cm}^{-1}$ was corresponding to the $\mathrm{A} 1 \mathrm{~g}$ mode of magnetite where peaks at 220 and 280 for Eg mode (Fig. 7a), these similar peaks were already reported (Justin et al. 2017; Mitchell et al. 2015; Panta and Bergmann 2015; Pham et al. 2016). There is a shift in Raman spectrum due to chitosan coating (Fig. 7b), which was also seen by Mai et al. (2012) where they coated chitosan on to magnetite. 

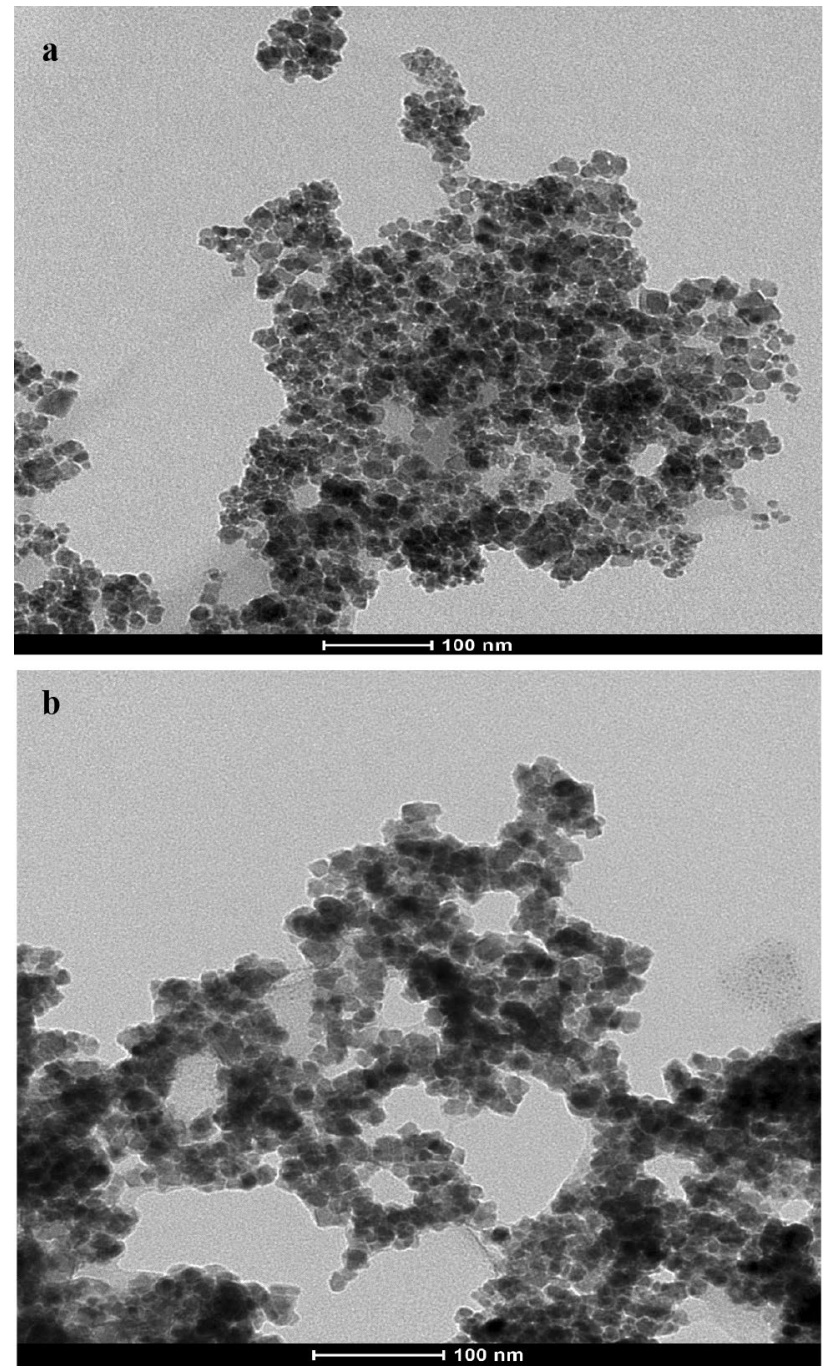

Fig. 3 TEM analysis of a SPIONs, $\mathbf{b}$ chitosan-coated SPIONs

\section{Chromium metal adsorption studies}

Chitosan-coated SPIONs have proven to be more efficient in the removal of chromium ions [Cr(VI)] from the solution (Fig. 8). The interaction between the amine groups of chitosan with the heavy metal had been already explained (Yavuz et al. 2006; Sarkar and Sarkar 2013), where the amines covalently bonds with heavy metal ions, thereby acting as an electron pair
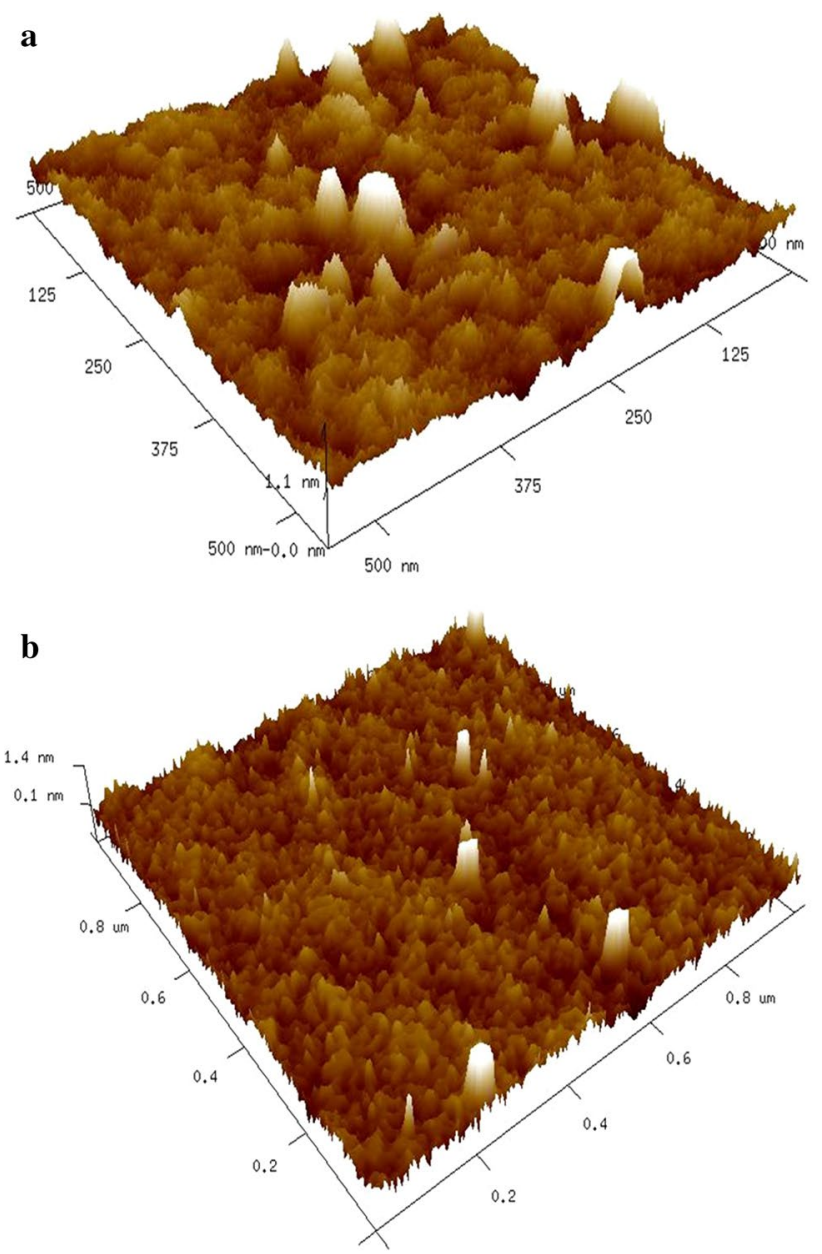

Fig. 4 AFM analysis of a SPIONs, b chitosan-coated SPIONs 
Fig. 5 X-ray diffraction analysis of a SPIONs, $\mathbf{b}$ chitosan-coated SPIONs
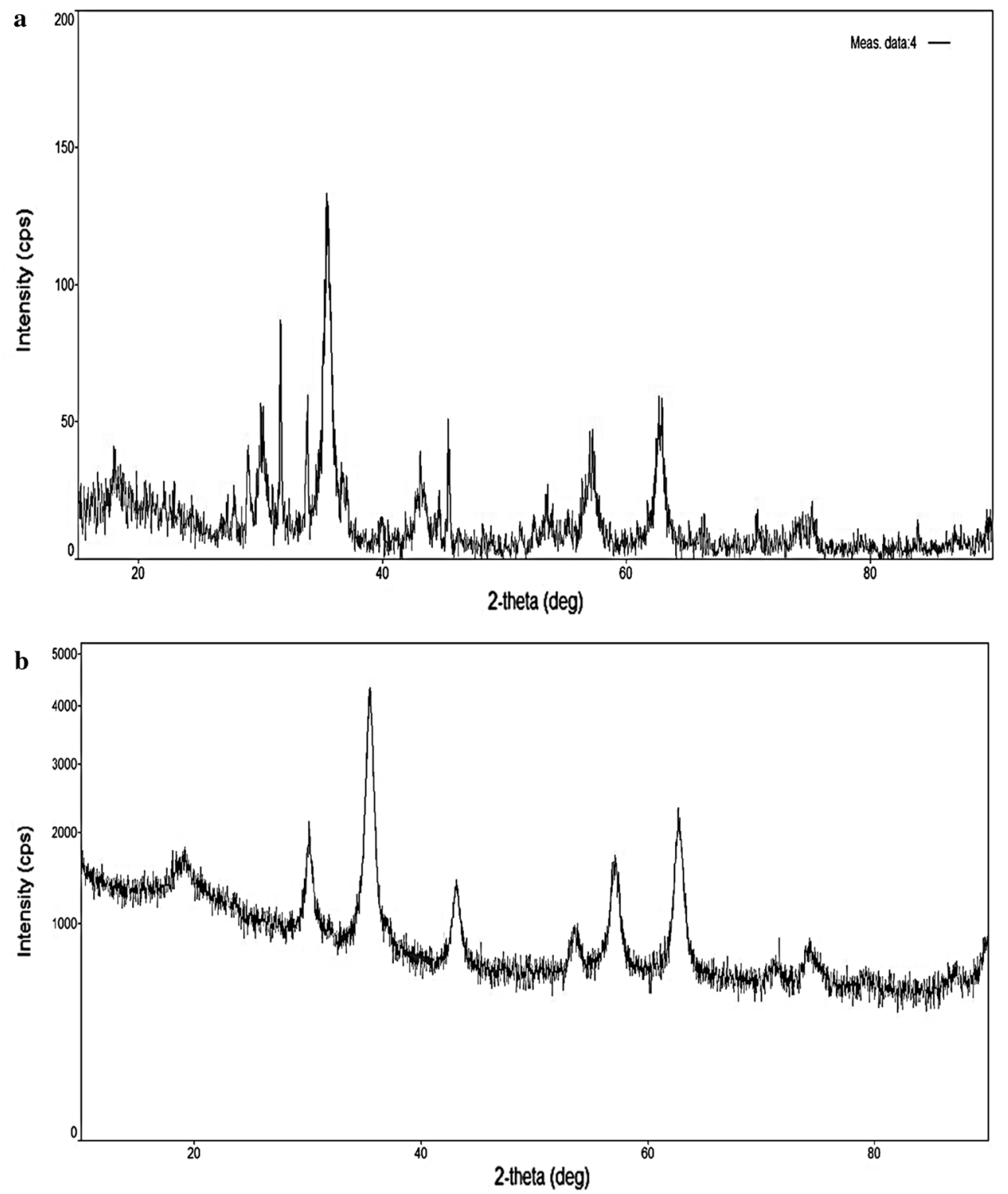

donor. Even the nanoparticles also adsorbs chromium; thus, chitosan-coated nanoparticles showed greater efficiency than the control. Even the applied acidic $\mathrm{pH}$ has its role in adsorption and removal of heavy metal from wastewater (Alzaidi et al. 2016; Shalaby et al. 2014).
In order to study the performance of adsorbent, which was chitosan-coated SPIONs, in the process of adsorption, different adsorption isotherms were plotted by deriving adsorption constants and analysing the $R^{2}$ values of each isotherm. The 
Fig. 6 Zeta potential analysis of a SPIONs, b chitosan-coated SPIONs a

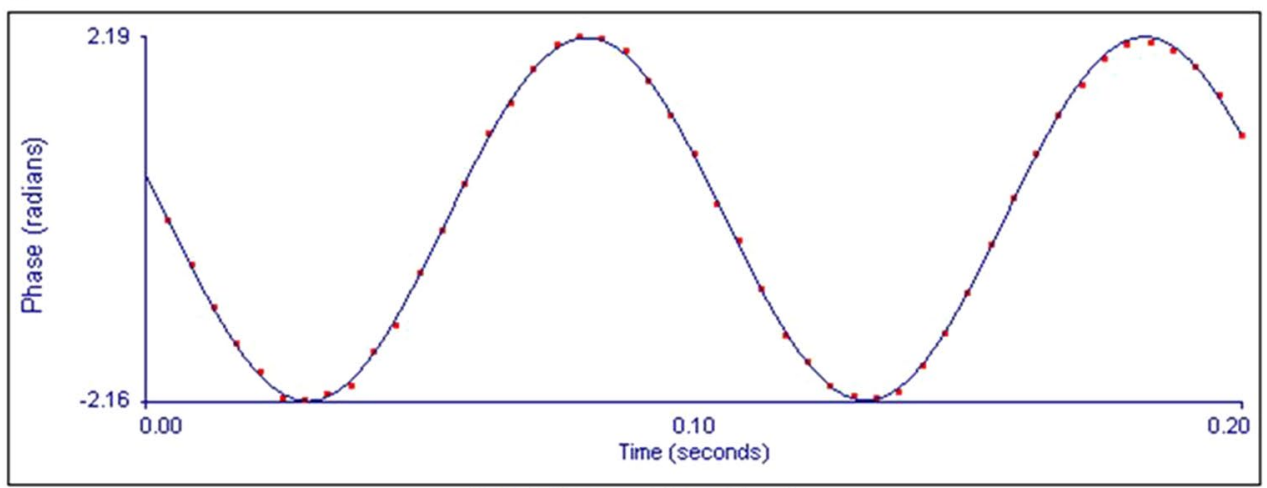

Fe304_2_repeat (Combined)

\begin{tabular}{|cccc|}
\hline Run & Mobility & Zeta Potential (mv) & Rel. Residual \\
\hline 1 & -2.98 & -38.10 & 0.0060 \\
2 & -2.98 & -38.19 & 0.0087 \\
& & & \\
& & & \\
& & & \\
& & & \\
& & & 0.0073 \\
Mean & -2.98 & & 0.0014 \\
Std. Error & 0.00 & 0.05 & 0.0051 \\
Combined & -2.98 & -38.14 & \\
\hline
\end{tabular}

b

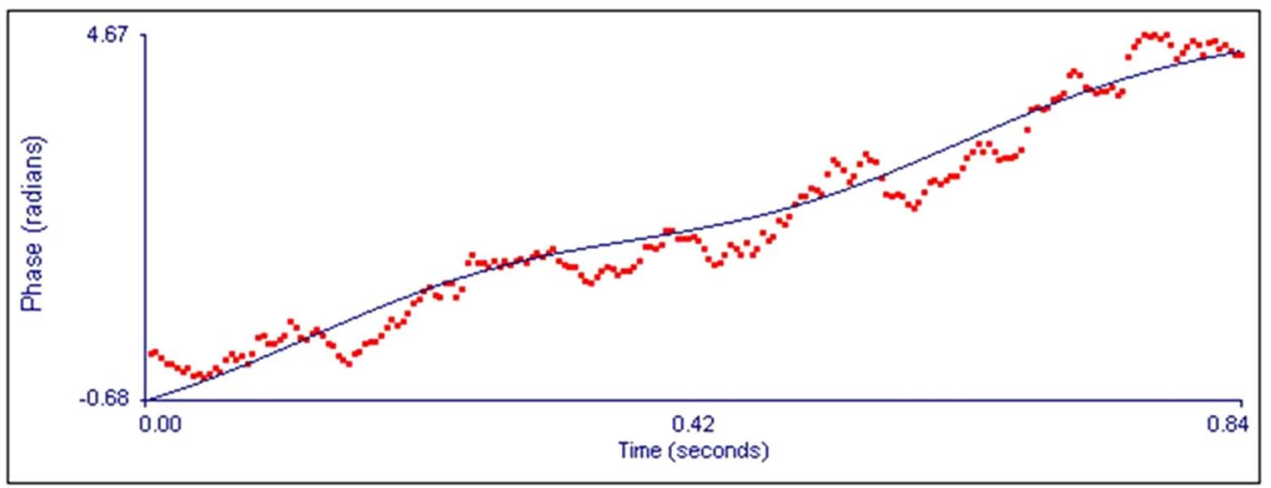

$\mathrm{P}$ (Combined)

\begin{tabular}{|cccc|}
\hline Run & Mobility & Zeta Potential (mv) & Rel. Residual \\
\hline 1 & 0.26 & 3.34 & 0.0285 \\
2 & 0.37 & 4.70 & 0.0176 \\
& & & \\
& & & \\
& & & \\
& & & \\
& & & 0.0230 \\
Mean & 0.31 & & 0.0055 \\
Std. Error & 0.05 & 0.68 & 0.0145 \\
Combined & 0.11 & 1.39 & \\
\hline
\end{tabular}


Fig. 7 Raman spectroscopy analysis of a SPIONs, $\mathbf{b}$ chitosan-coated SPIONs

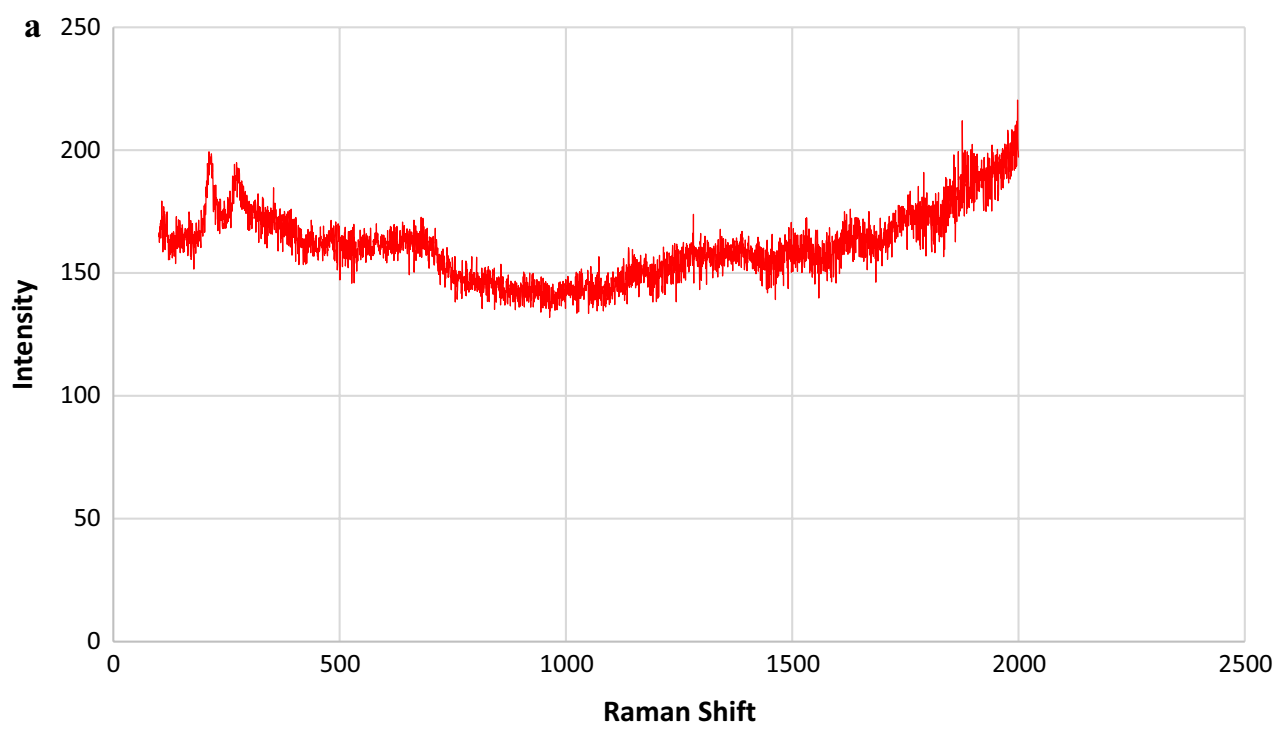

b

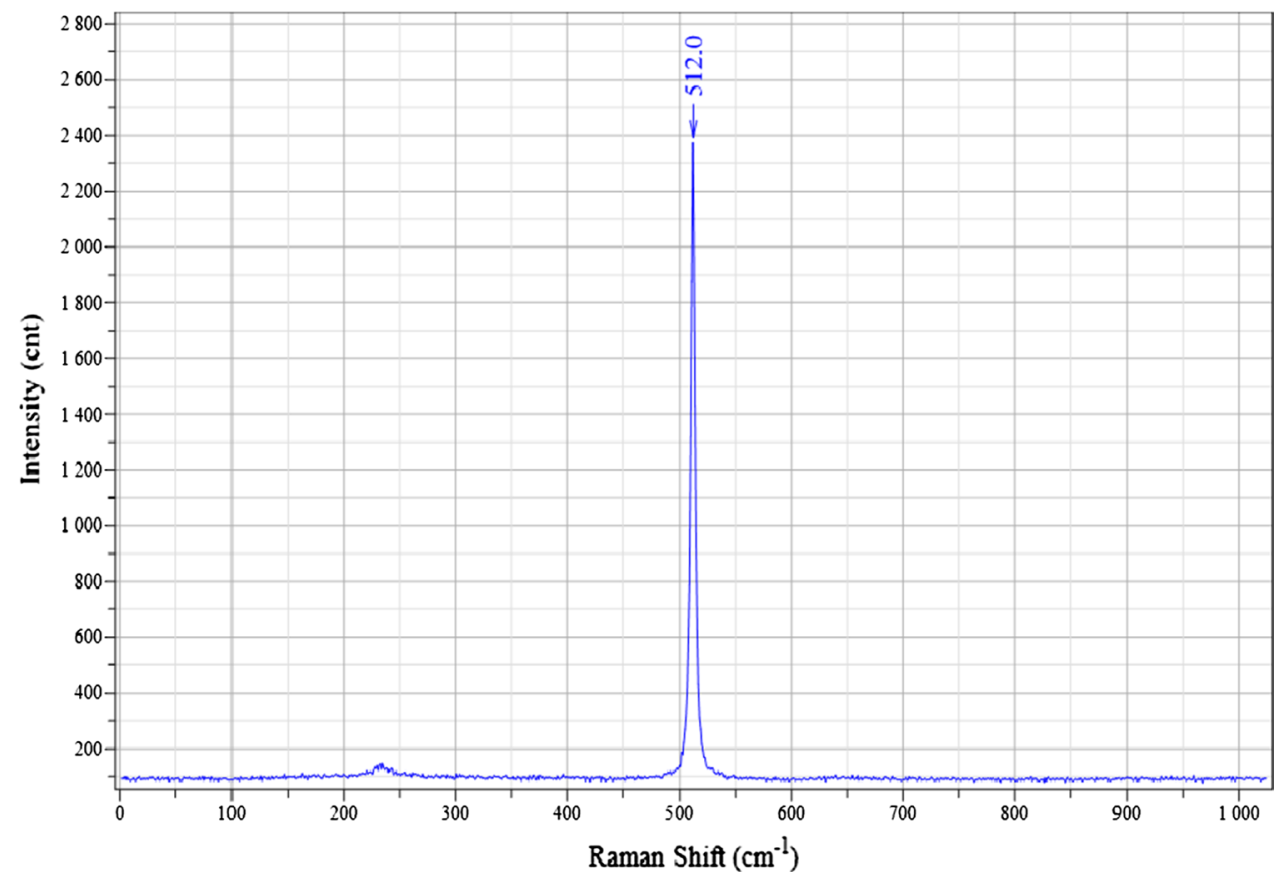

different isotherms plotted were Langmuir, Freundlich and Temkin isotherms. The comparison of $R^{2}$ of the linearized form of all the three adsorption equations indicated that the Freundlich model yields a better fit for the experimental equilibrium adsorption data with a value of 0.992 when compared to the other two (Fig. 9a-c). This suggested that the binding sites on the adsorbent were heterogeneous and multilayer adsorption of chromium could occur (Jiang et al. 2013; Shalaby et al. 2014).

\section{Summary and conclusions}

The chemical structure and the morphology of SPIONs and chitosan-coated SPIONs had been investigated. SPIONs coated with chitosan were interacting better with chromium and improved the heavy metal adsorption. The major reason must be the interaction between amine of chitosan and heavy metal leading to covalent bonding. Once the bonding is happening between the chitosan-coated SPIONs and heavy metal, removal of these SPIONs out of the water is easier as it 
Fig. 8 Percentage chromium removal efficiency of chitosancoated SPIONs at regular intervals of time

Fig. 9 Adsorption isotherm for chromium removal. a Langmuir isotherm. b Freundlich isotherm. c Tempkin isotherm
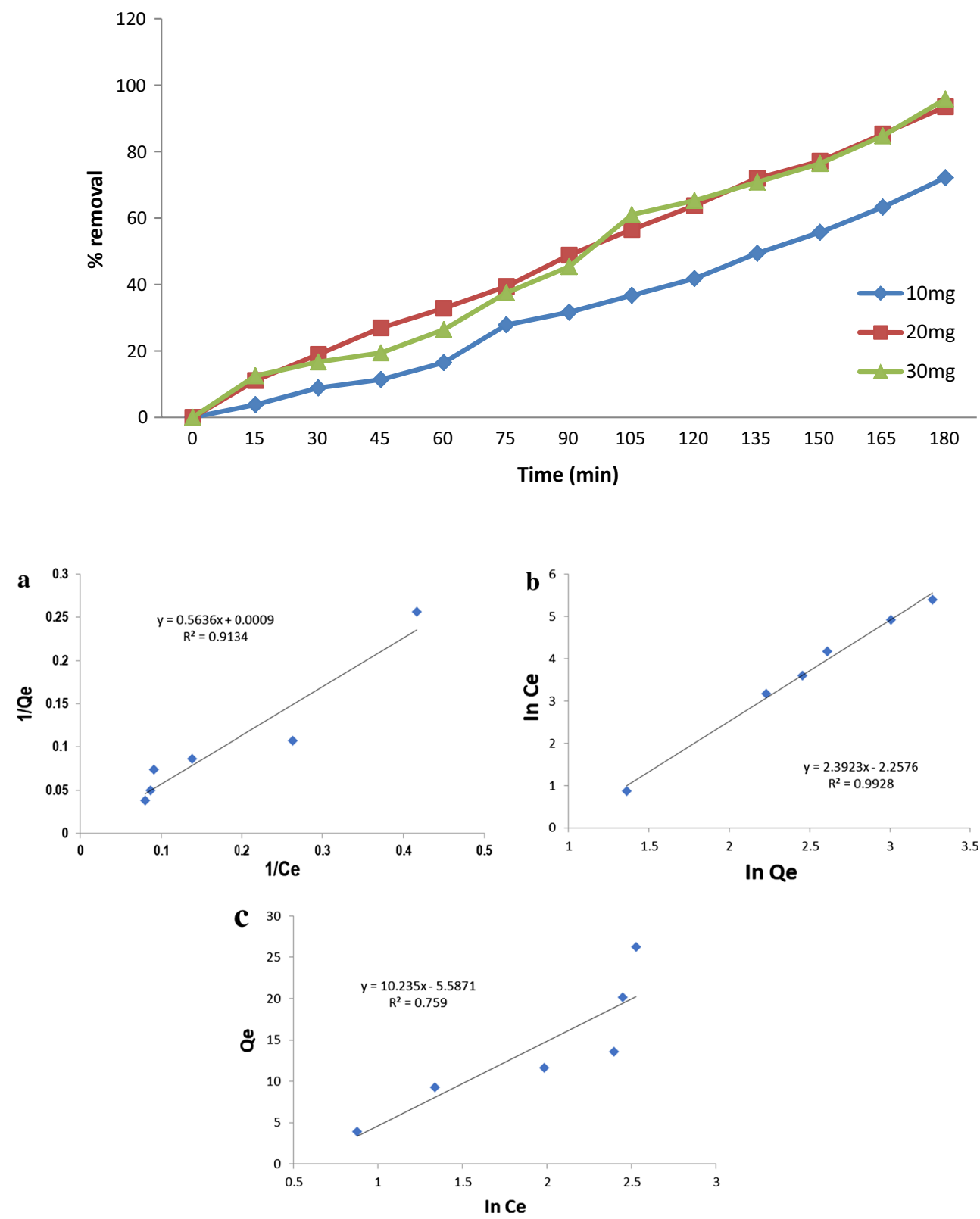

can be removed by applying external magnetic field. Thus, it can be employed for efficient removal of chromium ion from wastewater.

\section{Compliance with ethical standards}

Conflict of interest The authors declare no conflict of interest.

Open Access This article is distributed under the terms of the Creative Commons Attribution 4.0 International License (http://creativeco mmons.org/licenses/by/4.0/), which permits unrestricted use, distribution, and reproduction in any medium, provided you give appropriate credit to the original author(s) and the source, provide a link to the Creative Commons license, and indicate if changes were made.

\section{References}

Abdel-Raouf MS, Abdul-Raheim ARM (2017) Removal of heavy metals from industrial waste water by biomass-based materials: a review. J Pollut Eff Control 5:180

Ahluwalia SS, Goyal D (2007) Microbial and plant derived biomass for removal of heavy metals from wastewater. Bioresour Technol 98:2243-2257

Aitio A, Jarvisalo J, Kiilunen M, Kalliomaki PL, Kalliomaki K (1988) Chromium. In: Clarkson TW, Friberg L, Nordberg GF, Sager PR (eds) Biological monitoring of toxic metals. Plenum, New York, pp 369-382

Alzaidi J, Alzahrani E, El-Mouhty NRA (2016) Chemical studies on the preparation of magnetic nanoparticles coated with glycine and its application for removal of heavy metals. Orient J Chem 32(3):1503-1513 
Anah L, Astrini N (2017) Influence of pH on Cr(VI) ions removal from aqueous solutions using carboxymethyl cellulose-based hydrogel as adsorbent. IOP Conf Ser Earth Environ Sci 60(1):012010

Bhattacharjee S (2016) DLS and zeta potential-What they are and what they are not? J Control Release 235:337-351

Boddu V, Abburi K, Talbott JL, Smith E (2003) Removal of hexavalent chromium from wastewater using a new composite chitosan biosorbent. Environ Sci Technol 37:4449-4456

Dada AO, Olalekan AP, Olatunya AM, Dada O (2012) Langmuir, Freundlich, Temkin and Dubinin-Radushkevich isotherms studies of equilibrium sorption of $\mathrm{Zn}^{2+}$ unto phosphoric acid modified rice husk. IOSR J Appl Chem 3(1):38-45

Dayan AD, Paine AJ (2001) Mechanisms of chromium toxicity, carcinogenicity and allergenicity: review of the literature from 1985 to 2000. Hum Exp Toxicol 20(9):439-451

Eom TH, Tuan HT, Kim SJ, Suh DS (2010) Synthesis of iron oxide using ferrous and ferric sulfate. Korean J Mater Res 20(6):301-306

Geng B, Jin Z, Li T, Qi X (2009) Kinetics of hexavalent chromium removal from water by chitosan-FeO nanoparticles. Chemosphere $75: 825-830$

Gill SK, Singh G, Khatri M (2017) Synthesis and characterization of superparamagnetic iron oxide nanoparticles for water purification applications. Int J Eng Technol Sci Res 4(4):355-359

Inbaraj BS, Chen BH (2012) In vitro removal of toxic heavy metals by $\operatorname{poly}(\gamma$-glutamic acid)-coated superparamagnetic nanoparticles. Int J Nanomed 7:4419-4432. https://doi.org/10.2147/IJN.S34396

Jiang W, Pelaez M, Dionysiou DD, Entezari MH, Tsoutsou D, O'Shea K (2013) Chromium (VI) removal by maghemite nanoparticles. Chem Eng J 222:527-533

Justin C, Philip SA, Samrot AV (2017) Synthesis and characterization of superparamagnetic iron-oxide nanoparticles (SPIONs) and utilization of SPIONs in X-ray imaging. Appl Nanosci 7(7):463-475

Justin C, Samrot AV, Durga Sruthi P, Sahithya CS, Bhavya KS, Saipriya C (2018) Preparation, characterization and utilization of coreshell super paramagnetic iron oxide nanoparticles for curcumin delivery. PLoS ONE 13(7):e0200440

Kaveeshwar AR, Sanders M, Ponnusamy SK, Depan D, Subramaniam $\mathrm{R}$ (2018) Chitosan as a biosorbent for adsorption of iron (II) from fracking wastewater. Polym Adv Technol 29(2):961-969

Kumar KV, Sivanesan S (2006) Isotherm parameters for basic dyes onto activated carbon: comparison of linear and non-linear method. J Hazard Mater 129(1-3):147-150

Lasheen MR, El-Sherifa IY, Sabryb DY, El-Wakeela ST, El-Shahat MF (2013) Removal and recovery of $\mathrm{Cr}(\mathrm{VI})$ by magnetite nanoparticles. Desalination Water Treat 52(34-36): 1-10

Liu T, Wanga ZL, Zhaob L, Yanga X (2012) Enhanced chitosan/FeOnanoparticles beads for hexavalent chromium removal from wastewater. Chem Eng J 189:196-202

Mahmoudi M, Sant S, Wang B, Laurent S, Sen T (2011) Superparamagnetic iron oxide nanoparticles (SPIONs): development, surface modification and applications in chemotherapy. Adv Drug Deliv Rev 63(1-2):24-46

Mai TTT, Ha PT, Pham HN, Le TTH, Pham HL, Phan TBH, Tran DL, Nguyen XP (2012) Chitosan and O-carboxymethyl chitosan modified $\mathrm{Fe}_{3} \mathrm{O}_{4}$ for hyperthermic treatment. Adv Nat Sci Nanosci Nanotechnol 3:015006

Mane P, Bhosle AB, Jangam CM, Vishwakarma CV (2011) Bioadsorption of selenium by pretreated algal biomass. Adv Appl Sci Res 2:202
Marquez F, Herrera GM, Campo T, Cotto M, Duconge J, Sanz JM, Elizalde E, Perales O, Mornat P (2012) Preparation of hollow magnetite microspheres and their applications as drugs carriers. Nanoscale Res Lett 127:210

Mitchell E, De Souza F, Gupta RK (2015) Probing on the hydrothermally synthesized iron oxide nanoparticles for ultracapacitor applications. Powder Technol 272:295-299

Namasivayam C, Ranganathan K (1993) Waste Fe(III)/Cr(III) hydroxide as adsorbent for the removal of $\mathrm{Cr}(\mathrm{VI})$ from aqueous solution and chromium plating industry wastewater. Environ Pollut 82(3):255-261

Panta PC, Bergmann CP (2015) Raman spectroscopy of iron oxide of nanoparticles $\left(\mathrm{Fe}_{3} \mathrm{O}_{4}\right)$. J Mater Sci Eng 5:217. https://doi. org/10.4172/2169-0022.1000217

Pham XN, Nguyen TP, Pham TN, Tran TTN, Tran TVT (2016) Synthesis and characterization of chitosan coated magnetite nanoparticles and their application in curcumin drug delivery. Adv Nat Sci Nanosci Nanotechnol 7(4):045010. https://doi.org/10.1088/2043$6262 / 7 / 4 / 045010$

Piccin JS, Dotto GL, Pinto LAA (2011) Adsorption isotherms and thermochemical data of FD\&C Red $\mathrm{n} 40$ binding by chitosan. Braz J Chem Eng 28(2):295-304

Rajput S, Pittman CU Jr, Mohan D (2016) Magnetic magnetite $\left(\mathrm{Fe}_{3} \mathrm{O}_{4}\right)$ nanoparticle synthesis and applications for lead $\left(\mathrm{Pb}^{2+}\right)$ and chromium $\left(\mathrm{Cr}^{6+}\right)$ removal from water. Colloid Interface Sci 468:334-346

Samrot AV, Justin C, Padmanaban S, Burman U (2017) A study on the effect of chemically synthesized magnetite nanoparticles on earthworm: Eudrilus eugeniae. Appl Nanosci 7(1):17-23

Sarkar ZK, Sarkar FK (2013) Selective removal of lead (II) ion from wastewater using superparamagnetic monodispersed iron oxide $\left(\mathrm{Fe}_{3} \mathrm{O}_{4}\right)$ nanoparticles as a effective adsorbent. Int $\mathrm{J}$ Nanosci Nanotechnol 9(2):109-114

Shalaby TI, Fikrt NM, Mohamed MM, El Kady MF (2014) Preparation and characterization of iron oxide nanoparticles coated with chitosan for removal of $\mathrm{Cd}(\mathrm{II})$ and $\mathrm{Cr}(\mathrm{VI})$ from aqueous solution. Water Sci Technol 70(6):1004-1010

Sheet I, Kabbani A, Holail H (2014) Removal of heavy metals using nanostructured graphite oxide, silica nanoparticles and silica/ graphite oxide composite. Energy Proc 50:130-138

Sureshkumar V, Daniel KSCG, Ruckmani K, Sivakumar M (2016) Fabrication of chitosan-magnetite nanocomposite strip for chromium removal. Appl Nanosci 6:277-285

Tchounwou PB, Yedjou CG, Patlolla AK, Sutton DJ (2012) Heavy metal toxicity and the environment. EXS 101:133-164

Yavuz CT, Mayo JT, Yu WW, Prakash A, Falkner JC, Yean S, Cong L, Shipley HJ, Kan A, Tomson M, Natelson D, Colvin VL (2006) Low-field magnetic separation of monodisperse $\mathrm{Fe}_{3} \mathrm{O}_{4}$ nanocrystals. Science 314(5801):964-967

Yousafzai AM, Ullah F, Bari F, Raziq S, Riaz M, Khan K, Nishan U, Sthanadar IA, Shaheen B, Shaheen M, Ahmad H (2017) Bioaccumulation of some heavy metals: analysis and comparison of Cyprinus carpio and Labeo rohita from Sardaryab, Khyber Pakhtunkhwa. BioMed Research International, Cairo

Publisher's Note Springer Nature remains neutral with regard to jurisdictional claims in published maps and institutional affiliations. 